\title{
Obesity in menopause - our negligence or an unfortunate inevitability?
}

\author{
Jarosław Kozakowski, Małgorzata Gietka-Czernel, Dorota Leszczyńska, Agnieszka Majos
}

Department of Endocrinology, Centre of Postgraduate Medical Education, Bielański Hospital, Warsaw, Poland

\begin{abstract}
Numerous concerns about menopause exist among women, and fear of an increase in body weight is one of the most important of them. This paper presents an overview of current knowledge concerning the etiology of obesity related to menopause and about the mechanisms of its development, with particular regard to the hormonal changes that occur during this period of life. The role of estrogens in the regulation of energy balance and the effect of sex hormones on metabolism of adipose tissue and other organs are presented. The consequence of the sharp decline in the secretion of estrogens with subsequent relative hyperandrogenemia is briefly discussed. The main intention of this review is to clarify what is inevitable and what perhaps results from negligence and unhealthy lifestyles. In the last part of the paper the possibilities of counteracting the progress of adverse changes in body composition, by promoting beneficial lifestyle modifications and the use of hormonal substitution treatment, in cases where it is reasonable and possible, are described.
\end{abstract}

Key words: menopause, obesity, metabolic syndrome, cardiovascular risk.

\section{Introduction}

Menopause is usually a cause of many concerns among women. One of the most important is the fear of weight gain. In fact, it is well known that obesity and metabolic syndrome are found in women in this period of their life three times more often than before menopause [1]. However, there are a lot of myths - women are not sure whether obesity is associated with menopause per se, or perhaps with "hormonal therapy"? Is weight gain inevitable, something to which one should be reconciled, or is it the result of negligence in the areas of diet and physical activity?

Overweight and obesity according to the definition of the World Health Organization (WHO) are considered as an abnormal or excessive fat accumulation that may impair health (WHO, Obesity and overweight, fact sheet, updated June 2016). The Polish Society of Endocrinology (PSE) describes obesity as a chronic disease characterized by excessive accumulation of fat tissue, increasing the risk of so-called civilization diseases: cardiovascular, metabolic and cancers (Diagnostic and therapeutic algorithms of PSE, 2014).

Menopause is defined as occurring 12 months after the last menstrual period and marks the end of menstrual cycles. It is the result of a depleted pool of follicles in gonads and in consequence of the decline in estro- gen production. Menopause is preceded by a period of premenopause, when menses are still relatively regular, and a 3-12-month period of perimenopause with irregular bleeding. Among European (and Polish) women menopause appears currently on average at age $51.3 \pm 5$ years.

A lot of distressing symptoms in a woman's life appear during menopause, such as hot flashes, mood disorders, sleep disturbances, and recurring infections of the urogenital tract. Moreover, a lot of metabolic problems emerge at this time - increase in body weight, insulin resistance, and glucose and lipid metabolism disturbances. In consequence, the risk of development of type 2 diabetes, osteoporosis, cardiovascular and oncological diseases increases.

Obesity should be seen as one of the most important disturbances associated with the menopause, being not only a medical but also a social and economic problem. The incidence of obesity in the United States among women between 40 and 65 years is calculated as $65 \%$, and among women over the age of 65 as almost $74 \%$ [2]. Similar data relate to Polish women. Prevalence of metabolic syndrome (MS) after menopause, closely associated with obesity, is estimated at the level of $31-55 \%$, depending on the criteria of MS, socioeconomic and environmental differences as well as on genetic and ethnic factors [3]. 
The mechanisms of menopausal obesity. The role of estrogens in the regulation of metabolism and energy balance

The mechanisms of the increase in body weight, unfortunately usually in the form of central obesity, during menopause are still not clearly elucidated. The obvious cause seems to be the rapid fall in estrogen levels. However, the contribution of environmental factors should also be taken into account. The main causes of changes in body composition associated with menopause are summarized in Table I.

Estrogens in women are responsible for the accumulation of fat in the subcutaneous tissue, particularly in the gluteal and femoral regions. To cause a biological effect estrogens connect with receptors that belong to the family of nuclear receptors. There are two types of such receptors, ER $\alpha$ and $E R \beta$, which are in fact transcription factors that regulate expression of target genes. However, it is believed that in addition to the genomic action estrogens may also work in a non-genomic mechanism, depending on activation of specific receptors located on the cell membrane. Other sex hormones - androgens - promote the accumulation of abdominal fat. Hence, the development of obesity with metabolically unfavorable fat redistribution from gynoid to abdominal location observed during menopause is caused by the relative hyperandrogenemia in the conditions of lack of estrogens. Another important factor contributing to development of central obesity is a decrease in the hepatic production of sex-hormone binding globulin (SHBG), which increases the bioavailability of androgens. It was estimated that postmenopausal women had a 4.88 -fold higher risk of developing abdominal obesity compared to premenopausal subjects [4]. In return, obesity has an impact on the peripheral conversion of androgens into estrogens. As a result of the aromatization of testosterone and androstenedione to estrone $\left(E_{1}\right)$, estradiol $\left(E_{2}\right)$ and estriol $\left(E_{3}\right)$, levels of these hormones in obese postmenopausal women are higher than in nonobese subjects. In consequence, visceral fat mass increases during menopause by $44 \%$ and the mass of gynoid fat rises by approximately $32 \%$ [5]. Unfortunately, it seems that aromatase-derived estro- gens are not associated with the protective status for insulin sensitivity and cardiovascular diseases, and increased risk of type 2 diabetes, hypertension, and dyslipidemia, and a higher rate of cardiovascular diseases in postmenopausal women are observed.

\section{Sex hormones and metabolism of fat tissue}

Stimulation of ER $\alpha$ in adipose tissue affects metabolic activity of adipocytes. In general, ER $\alpha$ receptors are involved in the beneficial effect of estrogens on adipose tissue distribution, glucose metabolism, and inflammation [6]. However, fat tissue metabolism is in fact regulated primarily by the adrenergic system. Stimulation of the type $\beta$ adrenergic receptors increases lipolysis, while the activation of $\alpha 2 \mathrm{~A}$ receptors results in the antilipolytic action and contributes to the accumulation of fat. It has been shown that estradiol increases the expression of $\alpha 2 \mathrm{~A}$ receptors in subcutaneous fat tissue in women and does not affect these receptors in the visceral fat [7]. On the other hand, it was found that activation of the ER $\alpha$ receptors could stimulate adrenergic $\beta$ receptors in the visceral tissue, enhancing the process of lipolysis and in this way reducing abdominal fat mass. Moreover, estrogens increase fat oxidation in the skeletal muscles and inhibit hepatic and muscle lipogenesis [8]. So, it should be expected that the lack of estrogens associated with menopause would result in a tendency for an increase in the visceral fat mass. In turn, abdominal obesity is a key factor in the pathogenesis of insulin resistance and metabolic syndrome. Visceral fat mass is considered as a potential mediator between menopausal status and the degree of insulin resistance. However, the relationship between menopausal status and the decrease in insulin sensitivity is not entirely explained. It has been reported in some studies that there is a lack of direct effect of menopause on the development of insulin resistance [9, 10]. On the other hand, many authors have evidenced the existence of such relationship, although usually the study groups were not matched for BMI or waist circumference $[11,12]$. The problem of insulin sensitivity in postmenopausal women is of great importance, be-

Table I. Main factors contributing to menopausal changes in body composition

\begin{tabular}{ccc}
\hline Genetic factors & Hormonal factors & Exogenous factors \\
$\begin{array}{c}\text { Genetic predisposition } \\
\text { Ethnicity } \\
\text { Epigenetic changes }\end{array}$ & $\begin{array}{c}\text { Rapid hypoestrogenemia } \\
\text { Relative hyperandrogenemia } \\
\text { Low SHBG levels } \\
\text { Low physical activity } \\
\text { Drugs (e.g. steroids, insulin) } \\
\text { Diseases }\end{array}$ & $\begin{array}{c}\text { intion } \\
\text { Increase and redistribution of fat mass (from gynoid to abdominal obesity) } \\
\text { Decrease in fat-free mass }\end{array}$ \\
\hline
\end{tabular}


cause the most severe metabolic disturbances - high postprandial glucose, hypertriglyceridemia, high C-reactive protein and interleukin 6 (IL-6) levels as well as low levels of Apo A1 and HDL cholesterol - are found in postmenopausal women with both abdominal obesity and insulin resistance [13]. The relationship between degree of visceral obesity and insulin resistance is dependent not only on estrogens but also on the other sex hormones levels. Particularly free testosterone can induce insulin resistance both directly and indirectly through the increase of abdominal obesity [14]. Also low SHBG levels are an important factor contributing to insulin resistance development in postmenopausal women. Inversely, the elevated insulin stimulates the production of androgens in the mechanism of activation of cytochrome P450c17 $\alpha$, which is a key enzyme in the ovarian androgens' biosynthesis. Gonadotropins that are elevated in menopause are another important factor acting in mechanisms of androgen synthesis stimulation. However, it has been shown that estrogens administered to animals after ovariectomy normalize body weight, earlier increased as a result of the removal of the gonads, and restore insulin sensitivity, but only in the presence of ER $\alpha$ [15]. It confirms the important role of estrogens in the regulation of energy balance.

It is well known that fat tissue, particularly in obese subjects, is the source of many adipokines. One of the most important of them is leptin. Its levels reflect the volume of fat mass. Leptin inhibits appetite, acting centrally. It has been demonstrated that estrogens potentiate leptin action by increasing the expression and sensitivity of its receptors in the hypothalamus [16]. Also an inverse correlation between the levels of estrogens and adiponectin - another important cytokine - opposite to leptin, increasing insulin sensitivity, has been observed [17]. Moreover, estrogens inhibit the production of proinflammatory cytokines produced in fat tissue, such as IL-6 or tumor necrosis factor $\alpha$ (TNF- $\alpha$ ) [18].

Clinically important and measurable effects of sex hormones action on the metabolism of body fat are their impact on blood lipids levels. In postmenopausal women estrogens promote the maintenance of a favorable lipid profile, while the increase in androgen levels has an atherogenic effect [19].

Perimenopause is also the time in which women often experience an increase in blood pressure. In women older than 55 years of age hypertension is found more frequently than in men [20]. No similar relationship occurs in younger subjects. The pathogenesis of perimenopausal hypertension is complex. However, also in this case the role of estrogen deficiency, which leads to domination of vasoconstriction over vasodilatation effects, seems to be crucial. Lack of estrogens results in a decline in prostacyclin and nitric oxide levels [21]. Unfortunately, estrogen supplementation in physiological menopause does not reduce blood pressure signi- ficantly, indicating the contribution of other factors in the mechanism of hypertension development, e.g. the renin-angiotensin-aldosterone system. Also obesity with related insulin resistance and inflammation participate in the development of hypertension.

\section{Do estrogens affect appetite?}

Control of appetite and, more generally, of energy balance is continuously performed in the central nervous system (CNS), mainly in the hypothalamus. In this region nuclei - arcuate, ventro-medial and paraventricular - are located. Activation of specific neurons in these structures triggers an orexigenic or anorexigenic effect, i.e. stimulating or reducing appetite.

The expression of both types of estrogen receptors, mainly $E R \alpha$, occurs in this region. Activation of $E R \alpha$ on hypothalamic proopiomelanocortin (POMC) neurons inhibits appetite in experimental animals [22]. Also the expression of neuropeptide Y (NPY) - an orexigenic peptide produced in the hypothalamus - is inhibited in the presence of estrogens [23]. Moreover, activity of ghrelin - the strongest orexigenic peptide, derived mainly from the stomach, but also produced locally in the hypothalamus - is reduced by estrogens [24]. So overall it may be said that estrogens are involved in central regulation of energy balance, and act on the CNS to reduce appetite. It can be assumed that the rapid decline in levels of these hormones would contribute significantly to an increase in appetite.

Undoubtedly, weight gain during menopause is common. It is largely due to hormonal changes that take place at this time. However, also other factors should be taken into account. These include genetic as well as environmental factors, among which lifestyle changes (unhealthy diet, lack of physical activity), illness (e.g. Cushing disease, hypothyroidism), certain medications (e.g. corticosteroids, insulin, glitazones), and other factors should be mentioned.

Is an increase in body weight with its subsequent metabolic consequences in menopause inevitable? Is avoiding or reducing obesity possible?

Since the lack of estrogen is of crucial significance to the development of obesity and initiates the action of other involved factors, it may be considered that making up for the deficiency of these hormones, in the form of substitution therapy, would help to prevent an increase in body weight and normalize metabolic disturbances. Indeed, there is a lot of evidence that hormonal replacement therapy (currently called hormonal therapy of menopause [HTM]) reduces obesity, decreases abdominal fat mass (redistributes body fat), lowers the fasting glucose and insulin levels, improves blood lipid profile and, as a result, reduces cardiovascular risk $[25,26]$. The authors of a meta-analysis including more than 100 randomized controlled trials (RCT) concluded 
that HTM irrespective of the route of administration (orally or transdermal) and whether a progestin component was added to estrogens caused an increase in fatfree mass, decrease in visceral fat, increase in insulin sensitivity, and decrease in LDL/HDL cholesterol ratio, and $L p(a)$, fibrinogen and plasminogen activator inhibitor (PAI) levels. The impact of HTM on blood pressure was insignificant. In general, oral therapy had a more favorable effect on lipids and parameters of inflammation than transdermal therapy [27]. In another prospective RCT a decrease in fat mass, improvement in insulin sensitivity and lowering of blood pressure during HTM were observed [28].

It is quite obvious that HTM should be applied in accordance with the rules set out in the recommendations of scientific authorities, taking into account the indications and contraindications. Now it is known that treatment should be started during perimenopause or in the first few years after menopause. If initiated later it may even be counterproductive and harmful. There are more and more data on the desirability of long-term therapy, which should be aborted as a result of contraindications or lack of acceptance of, or inability to perform, control tests. It should be remembered that higher $E_{2}$ levels, not counterbalanced by progestins, might lead to massive bleeding in premenopausal women and to risk of hyperplasia and endometrial cancer after the menopause.

Not without significance is the attitude of the women themselves. Life after menopause can be a time dedicated to the development of their own interests, physical activity, reflection over the way of life and the implementation of healthy behaviors. Certainly, the cardiovascular events, hypertension, diabetes, hyperlipidemia, breast cancer risk, osteoporosis or urinary incontinence strongly depend on body weight, nutrition, physical activity and lifestyle decisions. The Framingham Nutrition Study showed that in women high calorie diet, rich in fat, containing alcohol with less fiber and micronutrients used for 12 years increases the risk of abdominal obesity and metabolic syndrome 3-4 fold [29]. On the other hand, a calorie-restricted diet with elimination of fast foods and carbohydrates but rich in sea fish, dietary products, fruits and vegetables is protective against obesity and metabolic disturbances [30].

Dietary modification should include a balanced, low-calorie diet. It is recommended to consume more fruits and vegetables and whole grains and reduce the intake of fats and carbohydrates. Although weight-loss programs recommend diets providing about $1500 \mathrm{cal}$, usually in proportions $60 \%$ carbohydrate, $30 \%$ fat, and $10 \%$ protein, the most important thing is to reduce the number of calories. As the lean body mass (including muscle mass) in menopause decreases, lower calorie intake in this period of life is needed. Eating while on one's feet, watching TV or playing games should be avoided. It is clear that the degree of weight loss is strongly dependent on the individual ability to adhere to dietary restrictions.

Recommendations of scientific societies in respect of lifestyle in the menopause emphasize the importance of combining diet with exercise. Limited physical activity during this time accelerates the progress of aging and increases the cardiovascular and osteoporosis risk.

Regular exercise (30 min/day, at least $2.5 \mathrm{~h} /$ week) is the best predictor for achieving long-term weight control and prevents cardiovascular events. Exercise habits added to diet help to promote greater weight loss. It has been proven that behavior that includes healthy eating and more intense physical activity in women in menopause is effective in reducing body weight, in particular the fat mass, whereas fat-free mass is maintained, and bone mass even increases.

\section{Conclusions}

Fear of obesity associated with menopause widely expressed among women seems to be justified. Observations confirm a high prevalence of weight gain and higher risk of many diseases related to obesity, especially cardiovascular events and cancers, in this period of women's life. Taking into account the growing frequency of overweight and obesity in the whole population, metabolic problems that appear in women in menopause should be considered as not only individual but also socioeconomic. However, gaining better understanding of the causes and mechanisms of weight gain during this time of life suggests that the occurrence of these unfavorable phenomena is not inevitable. They can be effectively countered by implementing the principles of a healthy lifestyle, and, when appropriate and possible, by hormonal therapy of menopause.

\section{Disclosure}

Authors report no conflict of interest.

\section{References}

1. Kwaśniewska M, Pikala M, Kaczmarczyk-Chałas K, et al. Smoking status, the menopausal transition, and metabolic syndrome in women. Menopause 2012; 19: 194-200.

2. Flegal KM, Carroll MD, Ogden CL, et al. Prevalence and trends in obesity among US adults, 1999-2008. J Am Med Ass 2010; 303: 235-241.

3. Stefańska A, Bergmann K, Sypniewska G. Metabolic Syndrome and Menopause: Patophysiology, Clinical and Diagnostic Significance. Adv Clin Chem 2015; 72: 1-75.

4. Donato GB, Fuchs SC, Oppermann K, et al. Association between menopause status and central adiposity measured at different cutoffs of waist circumference and waist-to-hip ratio. Menopause 2006; 13: 280285.

5. Panotopoulos G, Raison J, Ruiz JC, et al. Weight gain at the time of menopause. Hum Reprod 1997; 12 Suppl 1: 126-133.

6. Luglio HF. Estrogen and body weight regulation in women: the role of estrogen receptor alpha (ER- $\alpha$ ) on adipocyte lipolysis. Acta Med Indones 2014; 46: 333-338. 
7. Pedersen SB, Kristensen K, Hermann PA, et al. Estrogen controls lipolysis by up-regulating alpha2A-adrenergic receptors directly in human adipose tissue through the estrogen receptor alpha. Implications for the female fat distribution. J Clin Endocrinol Metab 2004; 89: 1869-1878.

8. Foryst-Ludwig A, Kintscher U. Metabolic impact of estrogen signalling through ERalpha and ERbeta.J Steroid Biochem Mol Biol 2010; 122: 74-81.

9. Toth MJ, Sites CK, Eltabbakh GH, et al. Effect of menopausal status on insulin-stimulated glucose disposal: comparison of middle-aged premenopausal and early postmenopausal women. Diabetes Care 2000; 23: 801-806.

10. Davis SR, Robinson PJ, Moufarege A, et al. The contribution of SHBG to the variation in HOMA-IR is not dependent on endogenous oestrogen or androgen levels in postmenopausal women. Clin Endocrinol (Oxf) 2012; 77: 541-547.

11. Carr MC. The Emergence of the Metabolic Syndrome with Menopause. J Clin Endocrinol Metab 2003; 88: 2404-2411.

12. Kalyani RR, Franco M, Dobs AS, et al. The Association of Endogenous Sex Hormones, Adiposity, and Insulin Resistance with Incident Diabetes in Postmenopausal Women. J Clin Endocrinol Metabl 2009; 94: 4127-4135.

13. Piché $M-E$, Weisnagel SJ, Corneau L, et al. Contribution of Abdominal Visceral Obesity and Insulin Resistance to the Cardiovascular Risk Profile of Postmenopausal Women. Diabetes 2005; 54: 770-777.

14. Sutton-Tyrrell K, Wildman RP, Matthews KA, et al. Sex-hormone-binding globulin and the free androgen index are related to cardiovascular risk factors in multiethnic premenopausal and perimenopausal women enrolled in the Study of Women Across the Nation (SWAN). Circulation 2005; 111: 1242-1249.

15. Barros RP, Gabbi C, Morani A, et al. Participation of ERalpha and ERbeta in glucose homeostasis in skeletal muscle and white adipose tissue. Am J Physiol Endocrinol Metab 2009; 297: E124-133.

16. Frank A, Lynda M. Brown LM, et al. The Role of Hypothalamic Estrogen Receptors in Metabolic Regulation. Front Neuroendocrinol 2014; 35: 550557.

17. Tworoger SS, Mantzoros C, Hankinson SE. Relationship of plasma adiponectin with sex hormone and insulin-like growth factor levels. Obesity (Silver Spring) 2007; 15: 2217-2224.

18. Monteiro R, Teixeira D, Calhau C. Estrogen Signaling in Metabolic Inflammation. Mediators Inflamm 2014; 2014: 615917

19. Shelley JM, Green A, Smith AM, et al. Relationship of endogenous sex hormones to lipids and blood pressure in mid-aged women. Ann Epidemiol 1998; 8: 39-45.

20. Maas AHEM, Franke HR. Women's health in menopause with a focus on hypertension. Neth Heart J 2009; 17: 68-72.

21. Reslan OM, Khalil RA. Vascular Effects of Estrogenic Menopausal Hormone Therapy. Rev Recent Clin Trials 2012; 7: 47-70.

22. Nestor CC, Kelly MJ, Rønnekleiv OK. Cross-talk between reproduction and energy homeostasis: central impact of estrogens, leptin and kisspeptin signaling. Horm Mol Biol Clin Investig 2014; 17: 109-128.

23. Rebouças EC, Leal S, Sá SI. Regulation of NPY and $\alpha-M S H$ expression by estradiol in the arcuate nucleus of Wistar female rats: a stereological study. Neurol Res 2016; 38: 740-747.

24. Dafopoulos K, Chalvatzas N, Kosmas G, et al. The effect of estrogens on plasma ghrelin concentrations in women. J Endocrinol Invest 2010; 33: 109-112.

25. Grodstein F, Manson JE, Colditz GA, et al. A prospective, observational study of postmenopausal hormone therapy and primary prevention of cardiovascular disease. Ann Intern Med 2000; 133: 933-941.

26. Schierbeck LL, Rejnmark L, Tofteng CL, et al. Effect of hormone replacement therapy on cardiovascular events in recently postmenopausal women: randomised trial. BMJ 2012; 345: e6409.

27. Salpeter SR, Walsh JM, Ormiston TM, et al. Meta-analysis: effect of hormone-replacement therapy on components of the metabolic syndrome in postmenopausal women. Diabetes Obes Metab 2006; 8: 538-554.

28. Samaras K, Hayward CS, Sullivan D, et al. Effects of postmenopausa hormone replacement therapy on central abdominal fat, glycemic control, lipid metabolism, and vascular factors in type 2 diabetes: a prospective study. Diabetes Care 1999; 22: 1401-1407.

29. Millen BE, Pencina MJ, Kimokoti RW, et al. Nutritional risk and the metabolic syndrome in women: opportunities for preventive intervention from the Framingham Nutrition Study. Am J Clin Nutr 2006; 84: 434-441.

30. Neuhouser ML, Howard B, Lu J, et al. A low-fat dietary pattern and risk of metabolic syndrome in postmenopausal women: the Women's Health Initiative. Metabolism 2012; 61: 1572-1581. 